\title{
Verzeichnis der Autoren und Herausgeber
}

Reinhard Altenhöner, Ständiger Vertreter der Generaldirektorin und Leiter der Zentralabteilung, Staatsbibliothek zu Berlin

Michaela Babion, Referatsleiterin Personal- und Organisationsentwicklung, Technische Informationsbibliothek, Hannover

Thomas Bähr, Leiter Bestandserhaltung und Langzeitarchivierung, Technische Informationsbibliothek, Hannover

Prof. Dr. Gabriele Beger, Direktorin, Staats- und Universitätsbibliothek Hamburg

Anke Berghaus-Sprengel, Direktorin, Universitäts- und Landesbibliothek Sachsen-Anhalt, Halle

Prof. Dr. Dagmar Blei, Vorstandsvorsitzende, Gesellschaft der Freunde und Förderer der SLUB

Dr. Achim Bonte, Stellvertreter des Generaldirektors, Sächsische Landesbibliothek - Staatsund Universitätsbibliothek Dresden

Dr. Jens Bove, Abteilungsleiter Deutsche Fotothek, Sächsische Landesbibliothek - Staats- und Universitätsbibliothek Dresden

Sabine Brünger-Weilandt, Direktorin und Geschäftsführerin, FIZ Karlsruhe - Leibniz-Institut für Informationsinfrastruktur $\mathrm{GmbH}$

Dr. Klaus Ceynowa, Generaldirektor, Bayerische Staatsbibliothek, München

Prof. Dr. Andreas Degkwitz, Direktor, Universitätsbibliothek der Humboldt-Universität zu Berlin Reiner Diedrichs, Direktor, Verbundzentrale des GBV (VZG), Göttingen

Prof. Dr. Frank Druffner, Kommissarischer Generalsekretär, Kulturstiftung der Länder

Prof. Dr. Dr. h. c. Bernhard Fabian, emer. Professor der Englischen Philologie und der Buchwissenschaft an der Westfälischen Wilhelms-Universität, Münster

Dr. Ralf Goebel, Direktor, Bibliotheksservice-Zentrum Baden-Württemberg, Konstanz

Dr. Thomas Haffner, Wissenschaftlicher Mitarbeiter, Abteilung Handschriften, Alte Drucke und Landeskunde, Sächsische Landesbibliothek - Staats- und Universitätsbibliothek Dresden

Dr. Ursula Hartwieg, Leiterin, Koordinierungsstelle für die Erhaltung des schriftlichen Kulturguts (KEK) an der Staatsbibliothek zu Berlin - Preußischer Kulturbesitz

Dr. Konstantin Hermann, Abteilungsleiter Bestandsentwicklung und Metadaten, Sächsische Landesbibliothek - Staats- und Universitätsbibliothek Dresden

Prof. Dr. Wolfram Horstmann, Direktor, Niedersächsische Staats- und Universitätsbibliothek Göttingen

Prof. Dr.-Ing. Matthias Kleiner, Präsident, Leibniz-Gemeinschaft

Dr. Michael Knoche, ehemals Direktor, Herzogin Anna Amalia Bibliothek, Weimar

Prof. Dr. h. c. Klaus-Dieter Lehmann, Präsident, Goethe-Institut

Dr. Anne Lipp, Gruppenleiterin Wissenschaftliche Literaturversorgungs- und Informationssysteme, Deutsche Forschungsgemeinschaft

Prof. Dr. Gilbert Lupfer, Leiter der Abteilung Forschung und wissenschaftliche Kooperation, Staatliche Kunstsammlungen Dresden

Prof. Dr. Claudia Lux, Project Director, Qatar National Library

Prof. Dr. Josef Matzerath, Professor für Sächsische Landesgeschichte, Technische Universität Dresden

Dr. Julia Meyer, Fachreferentin für Germanistik, Sächsische Landesbibliothek - Staats- und Universitätsbibliothek Dresden 
Prof. Dr.-Ing. habil. DEng/Auckland Hans Müller-Steinhagen, Rektor, Technische Universität Dresden

Martin Munke, Referatsleiter Saxonica, Sächsische Landesbibliothek - Staats- und Universitätsbibliothek Dresden

Dr. Dietrich Nelle, Interimsdirektor, Deutsche Zentralbibliothek für Medizin (ZB MED) - Informationszentrum Lebenswissenschaften, Köln/Bonn

Dr. Elisabeth Niggemann, Generaldirektorin, Deutsche Nationalbibliothek

Prof. Dr. Dr. h. c. mult. Hermann Parzinger, Präsident, Stiftung Preußischer Kulturbesitz

Prof. Dr. Roger Paulin, emer. Professor of German, University of Cambridge

Dr. Juliane Rehnolt, Wissenschaftliche Mitarbeiterin, Lehrstuhl für Neuere deutsche Literatur und Kulturgeschichte, Technische Universität Dresden

Prof. Dr. h. c. Klaus G. Saur, Verleger, München

Prof. Dr. Wolfgang Schmitz, ehemals Direktor, Universitäts- und Stadtbibliothek Köln

Prof. Dr. Ulrich Johannes Schneider, Direktor, Universitätsbibliothek Leipzig

Barbara Schneider-Kempf, Generaldirektorin, Staatsbibliothek zu Berlin - Preußischer Kulturbesitz

Hellmut Seemann, Präsident, Klassik Stiftung Weimar

Dr. Irina Sens, Stellvertretende Direktorin und Leiterin Bibliotheksbetrieb, Technische Informationsbibliothek, Hannover

Dr. Frank Simon-Ritz, Direktor, Universitätsbibliothek der Bauhaus-Universität Weimar

Konstanze Söllner, Direktorin, Universitätsbibliothek der FAU Erlangen-Nürnberg

Prof. Dr. Thomas Stäcker, Direktor, Universitäts- und Landesbibliothek Darmstadt

Dr. Eva-Maria Stange, Sächsische Staatsministerin für Wissenschaft und Kunst

Prof. Dr. Jochen Strobel, Professor für Neuere deutsche Literatur, Philipps-Universität Marburg

Prof. Dr. Peter Strohschneider, Präsident, Deutsche Forschungsgemeinschaft

Katrin Stump, Direktorin, Universitätsbibliothek der Technischen Universität Braunschweig

Dr. Michael Vogel, Abteilungsleiter Bestandserhaltung, Sächsische Landesbibliothek - Staats-

und Universitätsbibliothek Dresden

Dr. Andrea Wettmann, Direktorin, Sächsisches Staatsarchiv, Dresden

Dr. Barbara Wiermann, Abteilungsleiterin Musikabteilung, Sächsische Landesbibliothek Staats- und Universitätsbibliothek Dresden

Prof. Dr. Hans Wiesmeth, Präsident, Sächsische Akademie der Wissenschaften zu Leipzig 
